\title{
Effect of Paper and E-Dictionaries on Iranian EFL Learners' Reading Comprehension
}

\author{
Shiva Grami \\ Department of Foreign Languages, Isfahan (Khorasgan) Branch, Islamic, Azad University, Isfahan, Iran \\ E-mail: shiva.grami@yahoo.com \\ Mahmood Hashemian (Corresponding author) \\ Department of Foreign Languages, Isfahan (Khorasgan) Branch, Islamic Azad University, Isfahan, Iran \\ E-mail:m72h@hotmail.com
}

Received: $14-03-2017$

Published: 01-09-2017
Accepted: 07-05-2017

doi:10.7575/aiac.ijalel.v.6n.5p.199
Advance Access Published: July 2017

URL: http://dx.doi.org/10.7575/aiac.ijalel.v.6n.5p.199

\begin{abstract}
The purpose of this study was to investigate the effects of paper and e-dictionaries on Iranian intermediate learners' reading comprehension. To this end, 90 female English Foreign language learners were randomly selected and assigned into 2 experimental groups (e-dictionaries and paper dictionaries groups) and 1 control group. All the groups took a pretest using no dictionaries. After 2 weeks of treatment design for the experimental groups, all the 3 groups took part in the posttest. The experimental groups did their task with their relevant dictionaries, whereas the control group did their task without using any kind of dictionary. Data were analyzed through analysis of covariance (ANCOVA) and paired samples $t$ test. Results showed that the participants' reading comprehension improved from the pretest to the posttest in both experimental groups. Results also indicated that the learners in the e-dictionaries group outperformed those in the paper dictionaries group. The outcome of study reveals that e-dictionaries could improve students' reading comprehension by motivating them, shortening the time of searching words and reading a text, and increasing the number of look ups.
\end{abstract}

Keywords: E-dictionaries, Paper Dictionaries, Reading Comprehension

\section{Introduction}

Reading comprehension is one of the main aims of EFL teaching. Without comprehending the written text, English Foreign Language (EFL) learners cannot achieve academic progress from one learning stage to the other (Flynn, 2007).

Some researchers (e.g., Rizo-Rodríguez, 2004; Zarei \& Gujjar, 2012) believed that EFL teaching and learning not only can be done through different grammatical studies, but also they can benefit from a large range of teaching medium, among which dictionaries stand the most useful and the most widespread medium.

Up until lately, there were only the traditional paper dictionaries in the market. Nowadays, by the rapid development of technologies, online and e-dictionaries have found their place in the hands of many EFL learners because they are lighter, smaller, more stylish, and have colorful LCDs. They also contain millions of words that can be updated through internet only by a click (Zarei \& Gujjar, 2012).

In contrast to the enthusiasm EFL learners have towards e-dictionaries and see them as a preferable alternative to paper dictionaries, most EFL teachers seem to have negative attitudes towards e-dictionaries. However, both EFL teachers and learners have positive attitudes toward using e-dictionaries; EFL teachers do not agree about using e-dictionaries as a medium of English teaching (Dashtestani, 2014; Midlane, 2005; Tang, 1997). Several studies (e.g., Bataineh, 2014; Kobayashi, 2007) have been done to show the effect of e-dictionaries for EFL students' reading comprehension. Some L2 researchers (e.g., Bataineh, 2014; Osaki, Ochiai, Iso, \& Aizawa, 2003) believe that e-dictionaries help EFL students to learn language skills better than paper dictionaries. While others argue that there is no significant difference between e-dictionaries and paper dictionaries (e.g., Hamdi, 2015; Kobayashi, 2007; Koyama \& Takeuchi, 2007).

Meanwhile, Tang (1997) believed that e-dictionaries can cause distraction and less involvement with texts. They can also discourage EFL learners to learn through context. In addition, they make EFL learners antisocial because, instead of communicating with their classmates, they interact with machines in the classroom.

Dashtestani (2014) studied 126 EFL learners and 73 EFL teachers. They were all invited to respond to some questionnaires. He found out that despite the learners' general acceptance of e-dictionaries and moderately positive attitudes of both the teachers and learners toward the use of e-dictionaries for learning and L2, a great number of the teachers expressed their concerns over using e-dictionaries for learning an L2.

The current study was intended to investigate the effect of e-dictionaries and paper dictionaries on Iranian intermediate 
EFL learners' reading comprehension. This study had its origins in EFL teachers' and learners' point of view toward using different dictionaries and its effect on EFL learners' reading comprehension. Therefore, the present study was an attempt to particularly address the following research questions:

- Do e-dictionaries affect intermediate EFL learners' reading comprehension?

- Do paper dictionaries affect intermediate EFL learners' reading comprehension?

- Is there any significant difference between the effect of e-dictionaries and paper dictionaries on intermediate EFL learners' reading comprehension?

\section{Literature Review}

In recent decade, there has been growing interest in the use of e-dictionaries for EFL learning. Accordingly, there has been a rise in EFL learners' use of e-dictionaries for EFL purposes (Boonmoh, 2010; Nesi \& Haill, 2002).

Several studies have examined the efficacy of paper dictionaries and e-dictionaries consultation; many have shown that EFL learners' comprehension increases by looking up words through e-dictionaries (e.g., Bataineh, 2014; Flynn, 2007; Melhi, 2014), whereas others have resulted in no significant differences (e.g., Hamdi, 2015; Kobayashi, 2007; Koyama \& Takeuchi; 2007).

Flynn (2007) examines 174 intermediate Japanese EFL learners. He divides them into a group that read a text without dictionaries and groups that read the same text with either e-dictionaries or paper dictionaries. The results show that using dictionaries while reading a text result in more vocabulary gain and improve the participants' reading comprehension. In addition, using e-dictionaries enable the learners to achieve significantly higher scores on the reading comprehension test.

In 2014, Bataineh investigates the effect of e-dictionaries on reading comprehension on 43 EFL learners. He divides them into two groups: One read a text using multimedia (i.e., hypermedia annotations, glosses, and e-dictionaries) and the other group read the same text using paper dictionaries. He finds that e-dictionaries and other multimedia applications have a positive effect on the learners' reading comprehension.

Melhi (2014) examines 38 male EFL learners. He randomly assigns them into experimental and control groups. The learners in the experimental group read a text using e-dictionaries, whereas the learners in the other group read the same text without any kind of dictionary. The results show a statistically significant difference in favor of the experimental group.

Osaki, Ochiai, Iso, and Aizawa (2003) investigates the role of dictionary forms (paper and electronic) in finding the meaning of key words that were underlined in a text. It turns out that pocket e-dictionary consultation significantly facilitates choosing a contextually appropriate meaning and results in better reading comprehension scores than a reference to the paper dictionaries. The participants who are using the e-dictionaries spend much less time searching for the L2 vocabulary items than those consulting the paper dictionaries. Perhaps, the time saves by utilizing the edictionaries enable the learners to understand the context in more depth and, subsequently, increased student accuracy in searching for contextually appropriate meanings. The authors conclude that quick access to the information in the edictionaries may also have meant less interference in the process of reading that lead to the better comprehension scores.

On the other hand, by comparing the use of paper dictionaries, some L2 researchers have found that the use of edictionaries does not have advantages. There are L2 researchers who argue that there are no significant differences between paper and e-dictionaries (e.g., Hamdi, 2015; Koyama \& Takeuchi, 2003; Kobayashi, 2007).

Kobayashi (2007) has indicated that the tendency to use e-dictionaries results in an increase in dictionary consultation, which means more distractions and consecutively less comprehension. She collects the data through retrospective thinkaloud protocols and reading comprehension tests from $22 \mathrm{EFL}$ university learners.

Hamdi (2015) examines 44 EFL sophomores under two conditions: once using paper dictionaries and another time using e-dictionaries. He postulates that e-dictionaries enable the learners to read a text in a shorter period of time than paper dictionaries, but the type of dictionary does not influence the learners' comprehension of the text.

Koyama and Takeuchi (2007) study 34 Japanese EFL college learners into two experimental groups. They come to the conclusion that, however, the learners in the e-dictionary group fulfill their task in a shorter period of time and looked up more words than those in the paper dictionary group, but there is no significant difference between their reading comprehension scores.

\section{Method}

\subsection{Design}

The current study used a pretest-posttest control group design with 6 intermediate EFL classes which were randomly assigned to one of the 2 experimental groups and a control group. The students in the control group only engaged their regular classes without any treatment. They were not allowed to use any kind of dictionaries for doing their reading task, in the pretest, posttest, or at the class. The experimental groups received their relevant treatment for 2 weeks.

\subsection{Participants}

The participants were 90 Iranian female EFL intermediate learners at Pooyesh Language Institute in Isfahan, Iran. Their age ranged between 15 and 19 years old. The participants were selected from six language classes during two terms. Each term, three classes were involved in the study. Each class had 12 to 17 learners. They attended the class six days of 
a week, two hours a day. In each semester, the participants were randomly divided into three groups: a control group and two experimental groups (an e-dictionaries group and a paper dictionaries group).

In order to check the homogeneity of the participants in the experimental and control groups, the Oxford Placement Test (OPT; Allen, 1992) was administered to all the six classes. After calculating the mean scores, those learners whose scores were 1 standard deviation above and below the mean scores were selected as the participants.

\subsection{Materials and Instruments}

The first test was the OPT to assess the participants' degree of homogeneity prior to the study. Before and after the treatment, the participants were asked to do a pretest and a posttest, specifically designed to tap into their reading comprehension. They were provided with two texts to read - excerpted from Inside Reading 2 (Zwier, 2012). Each text contained 850 to 860 words and eight comprehension questions provided by the book in the true/false format.

The participants in the experimental groups were asked to have dictionaries with themselves in the class and at home. The participants in the paper dictionaries group were asked to use one of the standard widely used monolingual dictionaries, proper to their level of proficiency - intermediate or above - available in the markets such as the Oxford English Dictionary, Longman American Dictionary, and/or The American Heritage Dictionary of the English Language.

The participants in the e-dictionaries group were asked to use the e-version (e.g., Mobile application dictionaries, CDROM dictionaries, or online dictionaries) of the confirmed dictionaries, such as Oxford English Dictionaries, Longman American Dictionary, Merriam Webster Dictionary, English Collins Dictionary, Connect Cambridge Dictionary, and Electronic Pocket Dictionary. All the preceding dictionaries are based on the paper versions, but support more options than the paper ones.

\subsection{Procedure}

\subsubsection{Phase 1: Pretest}

For the pretest, the participants were asked to read a text without using any kind of dictionary. Then, they were asked to answer the comprehension tests below the text which consisted of eight statements about the text in English. Notwithstanding, their time laps was not the focus of the study; it was kept from the moment they had started to read the text for the moment they finished answering the test by one of the present researchers.

\subsubsection{Phase 2: Treatment and Introducing Dictionaries}

The participants of the e-dictionaries group were introduced with a variety of e-dictionaries (e.g., Mobile dictionary applications, online dictionaries, and e-pocket dictionaries), which are standard and verified by a university. They were taught how to install, search, listen, update or upgrade, tag, mark, star, and save a specific word.

The participants in the paper dictionaries group were introduced with different standard monolingual paper dictionaries. They were taught how to use the dictionaries and how to reach to the maximum speed of word checking.

The participants in the experimental groups were asked to use their dictionaries in the class and at home for both studying and doing their homework during two weeks in order to get familiar with their dictionaries to be ready for the posttest.

\subsubsection{Phase 3: Posttest}

After the treatment, the participants were given a posttest. The participants of the experimental groups were asked to read the text and check the meaning and the parts of speech of the words they doubted or did not know in their dictionaries as many times as they needed. Then, they were required to answer the comprehension questions below the text. During answering the comprehension test, the participants had the text and were allowed to check or recheck the words in order to minimize the effect of short-term memory (Flynn, 2007).

\subsubsection{Data Analysis}

The data were analyzed using the Statistical Package for the Social Science (SPSS, version 21.00). To provide answers for the research questions \# 1 and 2, the scores of the three groups on reading comprehension from the pretest and the posttest were submitted to paired samples $t$ test. One-way ANCOVA was conducted to find answers to research question \# 3. The post-hoc Scheffe test was run to find the exact location of the differences, if any.

\section{Results}

To find answers for the two first research questions, the pretest reading comprehension scores of the participants in the first experimental group (i.e., e-dictionary) were compared with their posttest reading comprehension scores via a paired samples $t$ test. Likewise, a paired samples $t$ test was conducted to compare the pretest and posttest reading comprehension scores of the participants in the second experimental group (i.e., paper dictionary). See Table 1 for the results:

Table 1. Descriptive Statistics of Pretests and Posttests Reading Comprehension Scores of Experimental Groups

\begin{tabular}{lllll}
\hline & Tests & $N$ & Mean & Standard Deviation \\
\hline Experimental Group \# 1 & Pretest & 30 & 4.03 & .66 \\
\cline { 2 - 5 } & Posttest & 30 & 7.10 & .71 \\
\hline Experimental Group \# 2 & Pretest & 30 & 3.50 & .50 \\
\cline { 2 - 5 } & Posttest & 30 & 4.70 & 1.29 \\
\hline
\end{tabular}


Comparing the mean scores of the reading comprehension pretest and posttest of both the experimental groups, it could be seen that the posttest mean scores were higher than the pretest mean scores for both groups. The significance or insignificance of these differences between the pretest and posttest scores of the experimental groups could be determined by examining the $\mathrm{p}$ value under the Sig. (2-tailed) column in Table 2:

Table 2. Paired Samples $t$-Test Results: Comparing Pretests and Posttests Reading Comprehension Scores of Experimental Groups

\begin{tabular}{llllllll}
\hline & & & Mean & Standard Deviation. & $t$ & $d f$ & $\begin{array}{l}\text { Sig. } \\
\text { (2-tailed) }\end{array}$ \\
\hline $\begin{array}{l}\text { Experimental Group } \\
\text { Pretest-Posttest }\end{array}$ & $\#$ & $1:$ & -3.06 & .52 & -32.25 & 29 & .000 \\
\hline $\begin{array}{l}\text { Experimental Group } \\
\text { Pretest-Posttest }\end{array}$ & $\#$ & $2:$ & -1.20 & 1.49 & -4.39 & 29 & .000 \\
\hline
\end{tabular}

For both experimental groups, the Sig. (2-tailed) column represents $p$ values lower than the significance level (.000 $<.05)$, indicating that the difference between the pretest and the posttest reading comprehension scores of the experimental groups were statistically significant. This is to say, both the e-dictionaries and paper dictionaries groups improved significantly from the reading comprehension pretest to the reading comprehension posttest (see Figure 1):

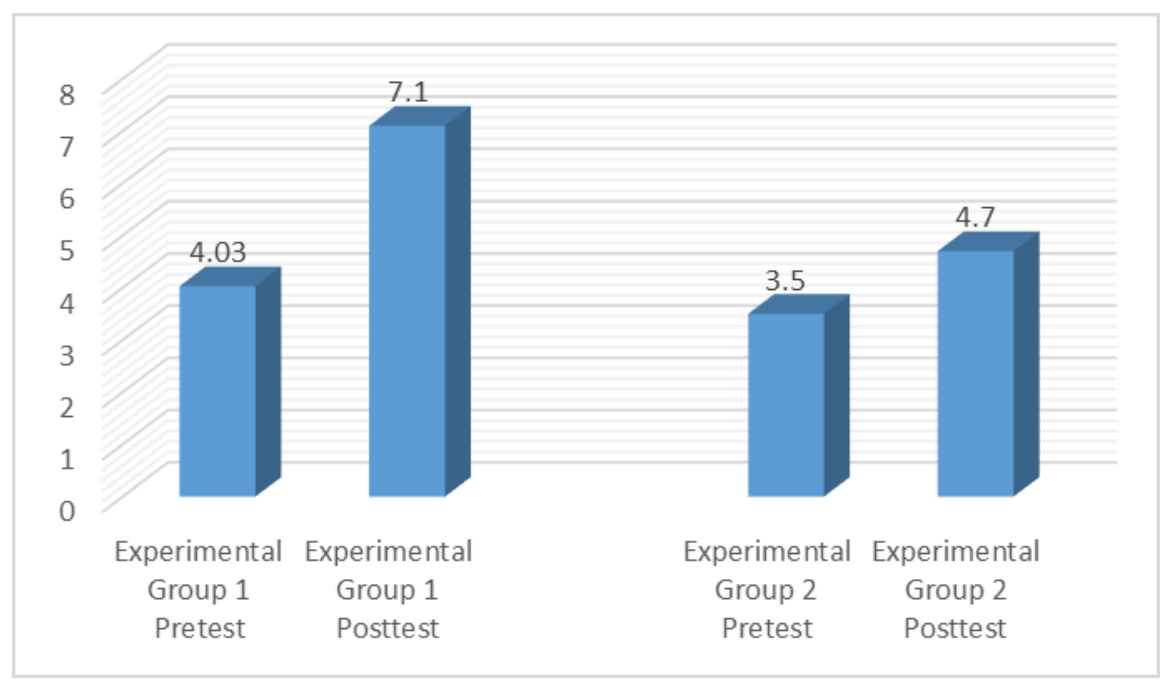

Figure 1. Mean Scores of Experimental Groups on Reading Comprehension Pretest and Posttest

It is clearly seen that the participants in the experimental groups showed a superior performance on the posttest, compared to the pretest. This indicates that the participants in the experimental groups' reading comprehension improved as a result of exposure to the treatment they had received.

To provide answer for the last research question, the posttest reading comprehension scores of the first experimental group, the second experimental group, and the control group had to be compared. Of course, attempts had to be made as to make sure these the three groups did not have preexisting differences. Hence, one-way ANCOVA was used to control for any possible preexisting differences among the three groups on the reading comprehension pretest and to compare their reading comprehension posttest performances (see Table 3):

Table 3. Descriptive Statistics for Comparing Posttest Reading Comprehension Scores of All Participants

\begin{tabular}{llll}
\hline Groups & Mean & Standard Deviation & $N$ \\
\hline Experimental Group \# 1 & 7.10 & .71 & 30 \\
\hline Experimental Group \# 2 & 4.70 & 1.29 & 30 \\
\hline Control Group & 3.80 & .84 & 30 \\
\hline Total & 5.20 & 1.70 & 90 \\
\hline
\end{tabular}

Such descriptive statistics as mean and standard deviation are shown for the three groups of the participants in Table 3 . The posttest reading comprehension mean score of the participants in the first experimental group $(M=7.10)$ was the highest mean score out there, and it was followed by the posttest reading comprehension mean score of the participants 
in the second experimental group $(M=4.70)$. The lowest mean score belonged to the control group $(M=3.80)$. To determine whether this difference among the three mean scores was a statistically significant one or not, one needed to look down the Sig (2-tailed) column in the Table 4:

Table 4. Results of One-Way ANCOVA for Comparing Posttest Reading Comprehension Scores of All Participants

\begin{tabular}{lllllll}
\hline Source & $\begin{array}{l}\text { Type III Sum } \\
\text { of Squares }\end{array}$ & $d f$ & Mean Square & $F$ & Sig. & Partial Eta Squared \\
\hline Corrected Model & 181.50 & 3 & 60.50 & 67.67 & .000 & .70 \\
\hline Intercept & 38.44 & 1 & 38.44 & 42.99 & .000 & .33 \\
\hline Pretest & 6.90 & 1 & 6.90 & 7.72 & .007 & .08 \\
\hline Groups & 153.08 & 2 & 76.54 & 85.60 & .000 & .66 \\
\hline Error & 76.89 & 86 & .89 & & & \\
\hline Total & 2692.00 & 90 & & & & \\
\hline Corrected Total & 258.40 & 89 & & & & \\
\hline
\end{tabular}

In Table 4, under the Sig. column, it can be seen that the $p$ value here is smaller than the specified level of significance $(.000<.05)$, indicating that there was a statistically significant difference among the three groups on the reading comprehension posttest.

The exact location of the difference should be found in the post-hoc Scheffe test table (Table 5), but before that, it is worth taking a second look at Table 4 under Partial Eta Squared, in front of Groups, the relevant value was .66. This means that being in one group or another accounted for $66 \%$ of the variance on the posttest reading comprehension scores of the participants. Another noteworthy piece of information in Table 4 concerns the influence of the covariate (i.e., the pretest). If you find the line in the table that corresponds to the covariate (i.e., the pretest) and read across to the Sig. level, you can see that the p value here is .007 , which is lower than the significance level, meaning that the covariate was significant, but it explained only $8 \%$ of the variance on the posttest reading comprehension scores of the participants (see Table 5):

Table 5. Results of Scheffe Post-Hoc Test for Comparing Posttest Reading Comprehension Scores of All Participants

\begin{tabular}{lllll}
\hline Groups & & Mean Difference & Standard Error Sig. \\
\hline Experimental Group \# 1 & Experimental Group \# 2 & $2.18^{*}$ & .25 & .000 \\
\cline { 2 - 5 } & Control Group & $3.19^{*}$ & .24 & .000 \\
\hline \multirow{2}{*}{ Experimental Group \# 2 } & Experimental Group \# 1 & $-2.18^{*}$ & .25 & .000 \\
\cline { 2 - 5 } & Control Group & $1.00^{*}$ & .24 & .000 \\
\hline \multirow{2}{*}{ Control Group } & Experimental Group \# 1 & $-3.19^{*}$ & .24 & .000 \\
\cline { 2 - 5 } & Experimental Group \# 2 & $-1.00^{*}$ & .24 & .000 \\
\hline
\end{tabular}

Pair-wise comparisons of the groups are presented in Table 5. As could be seen, the difference between the first experimental group $(M=7.10)$ and the second experimental group $(M=4.70)$ was statistically significant because the corresponding $\mathrm{p}$ value was found to be less than the significance level $(p=.000<.05)$. Similarly, the difference between the first experimental group and the control group $(M=3.80)$ was statistically significant. Moreover, the difference between the second experimental group and the control group reached statistical significance.

The conclusion to be made from the above analyses is that, on the test of reading comprehension, the group that had used e-dictionaries significantly outperformed the group that had used paper dictionaries, and that these two experimental groups were significantly superior to the control group.

\section{Discussion}

The results of this study tend to support the findings of Bataineh (2014), Flynn (2007), Korat and Shamir (2006), and Melhi (2014). They believed that using e-dictionaries while reading had positive effects on the EFL learners' reading comprehension. The results in this study indicated that the participants who had worked with e-dictionaries gained higher scores on the reading comprehension test than those who had used paper dictionaries or those who had not used dictionaries.

On the contrary, the outcome of this paper contradicts the findings of Hamdi (2015), Kobayashi (2007), as well as Koyama and Takeuchi (2007). Their findings illustrate that that there are no significant differences between paper dictionaries and e-dictionaries. 
This study revealed both e-dictionaries groups' and paper dictionaries group's test scores increased significantly from the reading comprehension pretest to the reading comprehension posttest, whereas the control group participants showed no significant improvement.

Before conducting this research, we had entertained a number of assumptions about the effect of paper and edictionaries on EFL learners' reading comprehension. These assumptions were as follows:

- $\mathrm{H}_{01}$ : E-dictionaries do not affect intermediate EFL learners' reading comprehension.

- $\mathrm{H}_{02}$ : Paper dictionaries do not affect intermediate EFL learners' reading comprehension.

- $\mathrm{H}_{03}$ : There is no significant difference between the effect of e-dictionaries and paper dictionaries on intermediate EFL learners' reading comprehension.

The results indicated that the scores of the participants who had used dictionaries, whether paper or electronic versions, improved from the reading comprehension pretest to the reading comprehension posttest. Therefore, both paper and edictionaries had statistically significant positive effects on the participants' reading comprehension. Thus, the null hypotheses \# 1 and 2 are rejected.

The results revealed that the participants in the e-dictionaries group significantly had better scores than the participants in the paper dictionaries group and the control group on reading comprehension posttest. This might be due the laps of time that the participants needed to finish their task. As we can see, the participants in the e-dictionaries group finished their task in less than $15 \mathrm{~min}$, whereas the participants in the paper dictionaries group did it in about $27 \mathrm{~min}$. According to Osaki et al. (2003), the time saved by the participants who had used e-dictionaries enabled them to process the text in more depth. They also claim that the short period of times means less interference in the reading process that leads to better comprehension scores. Arguably, the long laps of time cause fatigue. Therefore, EFL learners cannot involve in the process of reading deep enough to comprehend texts. According to Kobayashi (2007) the number of look-ups and EFL learners' tendency toward using e-dictionaries may effect and cause better and deeper comprehension. Thus, null hypothesis \# 3 is also rejected.

\section{Conclusion}

The current study aimed to investigate the effect of paper and e-dictionaries on Iranian EFL learners' reading comprehension. To that end, 90 female teenager EFL learners took participated in the study. They were divided into three groups: paper dictionaries group, e-dictionaries group, and control group. The experimental groups received their relevant treatment, which was introducing their relevant dictionaries and teaching the way of working with them. After that, the experimental groups took part in the posttest with their dictionaries and the control group did the posttest without any dictionary consultation.

The results indicated that both the paper dictionaries and e-dictionaries groups had significantly higher scores than those who had read without dictionaries on the posttest. Also, the e-dictionaries group scored significantly higher than the paper dictionaries group on the posttest.

The present study showed that e-dictionaries had positive effects on the participants' reading comprehension. The edictionaries group participants apparently outperformed the control group and the paper dictionaries group on the posttest. This is, to some extent, due to their higher speed of looking up the new words, their low weight, colorful LCD, and user- friendly features, such as favorite word list, history list, word games, and many others.

The paper dictionaries group considerably consumed more time than the e-dictionaries group which caused fatigue for their users. Consequently, they did not look up all the words that they did not know their meaning; they did not check the meaning of words they doubted; they did not use their dictionaries to find the parts of speech of new or even key words; they did not recheck the words they had found if they forgot its meaning and they did not check the words to see if it had other meanings beside the meaning(s) they knew; therefore, they failed to outperform the e-dictionaries group on the posttest.

Adaptation of this research to the L2 classroom will address multiple actions, including curriculum planning (i.e., the ways in which EFL reading instruction is planned), materials development (i.e., the ways in which reading tasks require L2 learners to check their dictionaries), and instructional decision making (i.e., the ways in which L2 teachers adopt technology in their class in order to teach reading to their learners).

As the participants of this study were teenage EFL students who were digital native, they grew up with so many different electronic gadgets, therefore they are more comfortable with e-dictionaries. At the end of this study, one may conclude that the fears of EFL teachers about students use of dictionary and their insisting on using paper dictionary in the classroom and at home (Dashtestani, 2014; Midlane, 2005) is unjustifiable.

Hence, the outcome of this examination confirm that the participants in the e-dictionaries group showed better performance than the other groups; equipping the classrooms and allowing L2 learners to use their e-dictionaries resulted in an increase in the reading comprehension scores. Moreover, the time that saved by using e-dictionaries could have been dedicated to extra practice and fun exercise that encouraged them to learn English.

Although this research was carefully prepared, there are still some limitations and shortcomings and the researchers are well aware of such shortcomings. This study was small scale: Only 30 learners in each group might not have represented the majority of EFL learners at the intermediate level. Thus, the result of this study cannot be generalizable.

Furthermore, the research was conducted in six intermediate classes during two summer terms. The process of treatment 
and observation lasted for two weeks, which was not enough for the researchers to observe all of the participants' performance in their classes. It would be better if it was done in a longer period of time.

The recommendations for further research include investigating the long-term effect of paper and e-dictionaries on EFL learners' reading comprehension. Further research can be a duplication of this study, with larger samples and in a more controlled situation. In addition, future researchers can conduct studies to determine the effectiveness of do-it-yourself dictionaries - the online dictionaries that made by users (e.g., Urban Dictionary and Wiktionary) — and their effect on EFL learners' learning skills.

\section{References}

Allen, D. (1992). Oxford placement test: Test pack. Oxford University Press.

Bataineh, A. M. (2014). The effect of electronic dictionaries and hypermedia annotations on English major learners' reading compression and vocabulary learning. International Journal of Linguistics, 6(4), 102-115.

Boonmoh, A. (2010). Teachers' use and knowledge of electronic dictionaries. ABAC journal, 30(3), 56-74.

Dashtestani, R. (2014). EFL teachers' perceptions of their computer literacy levels and challenges in improving EFL teachers' computer literacy. International Journal of Pedagogies \& Learning, 9(1), 51-65.

Flynn, M. H. (2007). Electronic dictionaries, printed dictionaries, and no dictionaries: The effects on vocabulary knowledge and reading comprehension. Unpublished doctoral dissertation, University of Birmingham, England.

Hamdi, C. (2015). The effects of electronic dictionary use on reading comprehension and vocabulary retention of EFL learners. Paper presented at Bejaia University International Conference (BUIC2015), Constantine University, Algeria.

Kobayashi, C. (2007). Comparing electronic and printed dictionaries: Their effects on lexical processing strategy use, word retention, and reading comprehension. In K. Bradford-Watts (Ed.), JALT 2006 conference proceedings (pp. 657671). Tokyo: JALT.

Koyama, T., \& Takeuchi, O. (2003). Printed dictionaries vs. electronic dictionaries: A pilot study on how Japanese EFL learners differ in using dictionaries. Language Education \& Technology, 40(1), 61- 79.

Koyama, T., \& Takeuchi, O. (2007). Does look-up frequency help reading comprehension of EFL learners? Two empirical studies of electronic dictionaries. Calico Journal, 25(1), 110-125.

Melhi, A. A. (2014). Effects on and predictability of computer-mediated glosses in reading comprehension of EFL college learners. Reading, 14(2), 65-77.

Midlane, V. (2005). Students' use of portable electronic dictionaries in the EFL/ESL classroom: A survey of teacher attitudes. Unpublished master's thesis, Faculty of Education, University of Manchester, Manchester, Britain.

Nesi, H., \& Haill, R. (2002). A study of dictionary use by international learners at a British University. International Journal of Lexicography, 15(4), 277-305.

Osaki, S., Ochiai, N., Iso, T., \& Aizawa, K. (2003). Electronic dictionary vs. printed dictionary: Accessing the appropriate meaning, reading comprehension, and retention. Paper presented at 3rd ASIALEX conference, Tokyo, Japan.

Rizo-Rodríguez, A. (2004). Current lexicographical tools in EFL: Monolingual resources for the advanced learner. Language Teaching, 37(1), 29-46.

Tang, G. (1997). Pocket electronic dictionaries for second language learning: Help or hindrance? TESL Canada Journal, 15(1), 39-57.

Zarei, A. A., \& Gujjar, A. A. (2012). The contribution of electronic and paper dictionaries to Iranian EFL learner's vocabulary learning. International Journal of Social Sciences \& Education, 2(4), 628-634.

Zwier, L. (2012). Inside reading 2: The academic word list in context (2 ${ }^{\text {nd }}$ ed.). New York: Oxford University Press. 